\title{
MIXED FINITE ELEMENT METHODS FOR COMPRESSIBLE MISCIBLE DISPLACEMENT IN POROUS MEDIA
}

\author{
SO-HSIANG CHOU AND QIAN LI
}

\begin{abstract}
A differential system describing compressible miscible displacement in a porous medium is given. The concentration equation is treated by a Galerkin method and the pressure equation is treated by a parabolic mixed finite element method. Optimal-order estimates in $L^{2}$ and almost optimal-order estimates in $L^{\infty}$ are obtained for the errors in the approximate solutions under the condition that $h_{p}^{2 k+2}\left(\log h_{c}^{-1}\right)^{1 / 2} \rightarrow 0$. This condition is much weaker than one given earlier by Douglas and Roberts for the same model. Furthermore, we obtain the $L^{\infty}\left(L^{2}(\Omega)\right)$-estimates for the time-derivatives of the concentration and the pressure, which were not given by the above authors. In addition, we also consider newer mixed spaces in two or three dimensions.
\end{abstract}

\section{INTRODUCTION}

We shall consider a two-component model for the single-phase, miscible displacement of one compressible fluid by another in a reservoir $\Omega \subset R^{2}$ of unit thickness. Let $c_{i}$ denote the concentration of the $i$ th component of the fluid mixture, $i=1,2$. Assume that the density $\rho_{i}$ of the $i$ th component and the pressure $p$ satisfy the equation of state in the form $d \rho_{i} / \rho_{i}=z_{i} d p$, where $z_{i}$ is the constant compressibility factor for the $i$ th component. The Darcy velocity of the fluid is given by $u=-\frac{k}{\mu} \nabla p$, where $k=k(x)$ is the permeability of the medium and $\mu=\mu\left(c_{1}, c_{2}\right)$ is the concentration-dependent viscosity. Let $D$ be a $2 \times 2$ matrix, $D=\varphi d_{m} I$, where $\varphi=\varphi(x)$ is the porosity of the rock, $I$ is the identity matrix, and $d_{m}$ is the coefficient of molecular diffusion. The model we consider is governed by the following differential system:

$$
\begin{aligned}
& \text { (a) } d(c) \frac{\partial p}{\partial t}+\nabla \cdot u=d(c) \frac{\partial p}{\partial t}-\nabla \cdot(a(c) \nabla p)=q, \\
& \text { (b) } \varphi \frac{\partial c}{\partial t}+b_{1}(c) \frac{\partial p}{\partial t}+u \cdot \nabla c-\nabla \cdot(D \nabla c)=(\hat{c}-c) q .
\end{aligned}
$$

Here,

$$
c=c_{1}=1-c_{2}, \quad a(c)=a(x, c)=k(x) \mu(c)^{-1},
$$

Received October 18, 1989; revised September 24, 1990.

1980 Mathematics Subject Classification (1985 Revision). Primary 65N30.

This research was supported in part by AFOSR Grant 88-0234. 


$$
\begin{gathered}
b_{1}(c)=b_{1}(x, c)=\varphi(x) c_{1}\left\{z_{1}-\sum_{j=1}^{2} z_{j} c_{j}\right\}, \\
d(c)=d(x, c)=\varphi(x) \sum_{j=1}^{2} z_{j} c_{j}, \\
q=\text { the external volumetric flow rate }
\end{gathered}
$$

and

$\hat{c}=$ the concentration of the 1 st component in the external flow.

We impose the no-flow conditions on the boundary:

(a) $u \cdot v=0 \quad$ on $\partial \Omega$,

(b) $(D \nabla c-c u) \cdot v=0$ on $\partial \Omega$,

where $v$ is the outer normal to $\partial \Omega$. In addition, the initial conditions are

(a) $p(x, 0)=p_{0}(x), \quad x \in \Omega$,

(b) $c(x, 0)=c_{0}(x), \quad x \in \Omega$.

The differential system (1.1) under the conditions (1.2) and (1.3) has been carefully derived by Douglas and Roberts [4], using sound physical reasoning. In [4], two numerical schemes for approximating the solution of the system (1.1)(1.3) were given. In both procedures the concentration equation (1.1b) was treated by a parabolic Galerkin procedure. In their second scheme the pressure equation was treated by a parabolic mixed finite element technique. The error analysis of the procedures was carried out under the assumptions that the solution is smooth, i.e., $q$ is smoothly distributed, the coefficients are smooth, and the domain has at least the regularity required for a standard elliptic Neumann problem to have $H^{2}(\Omega)$-regularity, and more, if the piecewise-polynomial spaces used in the finite element procedures have degree greater than one. Furthermore, the coefficients $a, d, \varphi$ are assumed to be bounded below positively.

The mixed space used in [4] is that of Raviart and Thomas. In this paper we also consider the newer spaces such as the BDM space [3] in two dimensions, the BDFM space [2], and the Nedelec space [8] in three dimensions.

Optimal $L^{2}$-estimates and quasi-optimal $L^{\infty}$-estimates for the errors have been given in [4] under the conditions that the maximum diameters $h_{c}$ and $h_{p}$ for the "concentration finite element space" and the "pressure finite element space," respectively, satisfy certain restrictions [4, relations (4.18) and (4.28)]. One of the purposes of this paper is to show that one can replace these restrictions by a much weaker condition (see (4.31) below) and at the same time obtain optimal $L^{2}$-estimates and quasi-optimal $L^{\infty}$-estimates. In addition, those optimal results hold for the newer spaces of $[2,3,8]$. Our analysis differs from that of [4] in one fundamental aspect. The mixed elliptic projection [4, equation 
(4.1)] is replaced by a nonlinear version of the elliptic projection presented in [6].

The rest of this paper is organized as follows. In $\S 2$, we give the weak formulations of the system (1.1)-(1.3) and discuss the associated finite element spaces. Section 3 is devoted to the error estimates for the concentration equation. The error estimates for the pressure equation are derived in $\S 4$, and the main results of the paper are contained in Theorem 4.1. In $\S 5$ we extend our results to three dimensions. A relation (5.1) relating discretization parameters is found, and optimal results are proved under this relation.

\section{WEAK FORMULATIONS AND FINITE ELEMENT APPROXIMATIONS}

We begin by putting (1.1b) into the following equivalent form:

$$
\varphi \frac{\partial c}{\partial t}-\nabla \cdot(D \nabla c)+u \cdot \nabla c-b(c) \nabla \cdot u=g(c)
$$

where $b(c)=b_{1}(c) d(c)^{-1}$. Let $(\cdot, \cdot)$ denote the inner product in $L^{2}(\Omega)$ or in $L^{2}(\Omega)^{2}$. Using Green's formula and (1.2), we have

$$
-(b(c) \nabla \cdot u, z)=(b(c) u, \nabla z)+\left(\frac{\partial b}{\partial c} u \cdot \nabla c, z\right)+\left(\frac{\partial b}{\partial x} \cdot u, z\right) .
$$

Thus, the weak form of (2.1) is to find a map $c: J \rightarrow H^{1}(\Omega)$ such that

$$
\begin{gathered}
\left(\varphi \frac{\partial c}{\partial t}, z\right)+(D \nabla c, \nabla z)+(b(c) u, \nabla z)+(e(c) u \cdot \nabla c, z) \\
\quad+(B(c) \cdot u, z)=(g(c), z), \quad z \in H^{1}(\Omega),
\end{gathered}
$$

where $e(c)=\frac{\partial b}{\partial c}(c)+1, B(c)=\frac{\partial b}{\partial x}=0$, and $J=[0, T]$.

Let $H(\operatorname{div}, \boldsymbol{\Omega})=\left\{v: v \in L^{2}(\boldsymbol{\Omega})^{2}, \nabla \cdot v \in L^{2}(\boldsymbol{\Omega})\right\}, V=\{v: v \in H(\operatorname{div}, \boldsymbol{\Omega})$, $v \cdot v=0$ on $\partial \Omega\}$, and $W=L^{2}(\Omega)$. Then the pressure equation (1.1a) is equivalent to the saddle point problem [4]: find a map $\{u, p\}: J \rightarrow V \times W$ such that

$$
\begin{array}{ll}
\text { (a) }\left(d(c) \frac{\partial p}{\partial t}, w\right)+(\nabla \cdot u, w)=(q, w), & w \in W, \\
\text { (b) }(\alpha(c) u, v)-(\nabla \cdot v, p)=0, & v \in V,
\end{array}
$$

where $\alpha(c)=a(c)^{-1}$.

To handle the nonzero initial conditions imposed on $p$ and $c$, we introduce the following transformations:

$$
\begin{aligned}
c(x, t) & =c^{*}(x, t)+c_{0}(x), \quad p(x, t)=p^{*}(x, t)+p_{0}(x), \\
u(x, t) & =\left[-a\left(c^{*}+c_{0}\right) \nabla\left(p^{*}+p_{0}\right)+a\left(c_{0}\right) \nabla p_{0}\right]-a\left(c_{0}\right) \nabla p_{0} \\
& =u^{*}(x, t)+u_{0}(x) .
\end{aligned}
$$


The reason for introducing these transformations is to validate equations (3.16) and (4.22) below. Now (2.2) can be written as

$$
\begin{aligned}
& \left(\varphi \frac{\partial c^{*}}{\partial t}, z\right)+\left(D \nabla c^{*}, \nabla z\right)+\left(b^{*}\left(c^{*}\right) u^{*}, \nabla z\right)+\left(b^{*}\left(c^{*}\right) u_{0}, \nabla z\right) \\
& \quad+\left(e^{*}\left(c^{*}\right) u^{*} \cdot \nabla c^{*}, z\right)+\left(e^{*}\left(c^{*}\right) u_{0} \cdot \nabla c^{*}, z\right)+\left(A^{*}\left(c^{*}\right) \cdot u^{*}, z\right) \\
& \quad=\left(f^{*}\left(c^{*}\right), z\right)-\left(D \nabla c_{0}, \nabla z\right), \quad z \in H^{1}(\Omega),
\end{aligned}
$$

where

$$
\begin{aligned}
& b^{*}\left(c^{*}\right)=b\left(c^{*}+c_{0}\right), \quad e^{*}\left(c^{*}\right)=e\left(c^{*}+c_{0}\right), \\
& A^{*}\left(c^{*}\right)=B\left(c^{*}+c_{0}\right)+e\left(c^{*}+c_{0}\right) \nabla c_{0}, \\
& f^{*}\left(c^{*}\right)=g\left(c^{*}+c_{0}\right)-\left[e\left(c^{*}+c_{0}\right) \nabla c_{0}+B\left(c^{*}+c_{0}\right)\right] \cdot u_{0} .
\end{aligned}
$$

Similarly, (2.3) can be expressed as

$$
\begin{gathered}
\text { (a) }\left(d^{*}\left(c^{*}\right) \frac{\partial p^{*}}{\partial t}, w\right)+\left(\nabla \cdot u^{*}, w\right)=(r, w), \quad w \in W, \\
\text { (b) }\left(\alpha^{*}\left(c^{*}\right) u^{*}, v\right)-\left(\nabla \cdot v, p^{*}\right) \\
=\left(\nabla \cdot v, p_{0}\right)-\left(\alpha^{*}\left(c^{*}\right) u_{0}, v\right), \quad v \in V,
\end{gathered}
$$

where

$$
d^{*}\left(c^{*}\right)=d\left(c^{*}+c_{0}\right), \quad \alpha^{*}\left(c^{*}\right)=\alpha\left(c^{*}+c_{0}\right), \quad r=q-\nabla \cdot u_{0} .
$$

The initial conditions for $c^{*}, p^{*}$, and $u^{*}$ can easily be seen to be

$$
\begin{aligned}
& \text { (a) } c^{*}(x, 0)=0, \\
& \text { (b) } p^{*}(x, 0)=0, \\
& \text { (c) } u^{*}(x, 0)=0 .
\end{aligned}
$$

Consequently, solving (1.1)-(1.3) is equivalent to solving (2.5)-(2.7). For ease of notation, we shall drop the superscript ${ }^{*}$ in (2.5)-(2.7) for the time being.

Let $h=\left(h_{c}, h_{p}\right)$, where $h_{c}$ and $h_{p}$ are positive. Let $M_{h}=M_{h_{c}} \subset W^{1, \infty}(\Omega)$ be a standard finite element space associated with a quasi-regular polygonalization of $\Omega$ and piecewise polynomials of some fixed degree not exceeding $l$. Thus, all standard inverse relations hold on $M_{h}$ and the approximation property

$$
\inf _{z_{h} \in M_{h}}\left\|z-z_{h}\right\|_{1, q} \leq M h_{c}^{l}\|z\|_{l+1, q}, \quad z \in W^{l+1, q}(\Omega), \quad 1 \leq q \leq \infty,
$$

holds, where $\|z\|_{k, q}$ is the norm in the Sobolev space $W^{k, q}(\Omega)$. In the following we also use $\|z\|_{k}$ for $\|z\|_{k, 2}$ and $\|z\|$ for $\|z\|_{0,2}$.

Suppose that $\Omega$ is a polygonal domain. Given a quasi-uniform decomposition of $\Omega$ into triangles or rectangles of diameter less than $h$, there exist $V_{h}=V_{h}^{k} \subset V$ and $W_{h}=W_{h}^{k} \subset W$ for $k \geq 0$ such that the elements have diameters bounded by $h_{p}$. The boundary condition $v \cdot v=0$ on $\partial \Omega$ is imposed 
on $V_{h}$. Assume that the following approximation properties hold:

(a) $\inf _{v_{h} \in V_{h}}\left\|v-v_{h}\right\|=\inf _{v_{h} \in V_{h}}\left\|v-v_{h}\right\|_{L^{2}(\Omega)^{2}}$

$$
\leq M h_{p}^{m}\|v\|_{m}, \quad 1 \leq m \leq k+1
$$

(b) $\inf _{v_{h} \in V_{h}}\left\|\nabla \cdot\left(v-v_{h}\right)\right\| \leq M h_{p}^{m}\left(\|v\|_{m}+\|\nabla \cdot v\|_{m}\right), \quad 1 \leq m \leq k$,

(c) $\inf _{w_{h} \in W_{h}}\left\|w-w_{h}\right\| \leq M h_{p}^{m}\|w\|_{m}, \quad 1 \leq m \leq k$.

The spaces $V_{h}^{k}$ and $W_{h}^{k}$ can be the spaces of Raviart and Thomas [10] for $k \geq 0$, or the spaces of Brezzi, Douglas, and Marini [3] for $k \geq 1$. If the Raviart-Thomas spaces are used, (2.9b) and (2.9c) are also valid for $m=k+1$. The discrepancy in the range of the index $m$ makes any attempt at handling the two spaces of the same index $k$ simultaneously rather awkward. Hence, we will carry out the error analysis only for the Raviart-Thomas space of index $k$. From now on we shall assume $V_{h} \times W_{h}$ to be the space of [10]. To obtain results for the BDM space of [3], one needs only to replace every occurrence of $k$ by $k-1$ in Theorem 4.1. This is so because the error analysis below depends only on (2.9) and (4.6) (with obvious modifications on the range of the index when the BDM space is used). Hence, any result in Theorem 4.1 obtained for the Raviart-Thomas space of index $k$ is also valid for the BDM space of index $k-1$. In other words, the error analysis for these two spaces are identical.

The semidiscrete finite element approximation for problem (2.5)-(2.7) amounts to finding a map $\{C, U, P\}: J \rightarrow M_{h} \times V_{h} \times W_{h}$ such that

$$
\begin{aligned}
& \text { (a) }\left(\varphi \frac{\partial C}{\partial t}, z\right)+(D \nabla C, \nabla z)+(b(C) U, \nabla z) \\
& +\left(b(C) u_{0}, \nabla z\right)+(e(C) U \cdot \nabla C, z) \\
& +\left(e(C) u_{0} \cdot \nabla C, z\right)+(A(C) \cdot U, z) \\
& =(f(C), z)-\left(D \nabla c_{0}, \nabla z\right), \quad z \in M_{h}, \\
& \text { (bi) }\left(d(C) \frac{\partial P}{\partial t}, w\right)+(\nabla \cdot U, w)=(r, w), \quad w \in W_{h} \text {, } \\
& \text { (bii) }(\alpha(C) U, v)-(\nabla \cdot v, P) \\
& =\left(\nabla \cdot v, p_{0}\right)-\left(\alpha(C) u_{0}, v\right), \quad v \in V_{h}, \\
& \text { (c) } C(0)=0, \quad P(0)=0 \text {. }
\end{aligned}
$$

From (2.6) and (2.7), we have

$$
\left(\nabla \cdot v, p_{0}\right)-\left(\alpha(0) u_{0}, v\right)=0, \quad v \in V .
$$

Then, setting $t=0$ in (2.10bii) and using (2.10c) and (2.11), we obtain

$$
U(0)=0 \text {. }
$$

\section{ERROR ESTIMATES FOR THE CONCENTRATION EQUATION}

In the analysis below, all functions of $c$ are assumed to be extended outside $[0,1]$ in some smooth fashion. 
Let us introduce an elliptic projection of the solution of (2.5) onto $M_{h}$, which is a map $\bar{c}: J \rightarrow M_{h}$ defined by

$$
\begin{aligned}
& (D \nabla(\bar{c}-c), \nabla z)+\left((e(c) \nabla(\bar{c}-c)) \cdot\left(u+u_{0}\right), z\right) \\
& \quad+\lambda(\bar{c}-c, z)=0, \quad z \in M_{h},
\end{aligned}
$$

where $\lambda$ will be selected large enough to ensure the coerciveness of the bilinear form on $H^{1}(\Omega)$. By standard results on the finite element method for elliptic problems, and by $[5,7,12,13]$, we know that

$$
\begin{gathered}
\text { (a) }\|\bar{c}-c\|+\left\|\frac{\partial(\bar{c}-c)}{\partial t}\right\|+\left\|\frac{\partial^{2}}{\partial t^{2}}(\bar{c}-c)\right\|+h_{c}\|\nabla(\bar{c}-c)\| \\
\leq M h_{c}^{l+1}\left(\|c\|_{l+1}+\left\|\frac{\partial c}{\partial t}\right\|_{l+1}+\left\|\frac{\partial^{2} c}{\partial t^{2}}\right\|_{l+1}\right), \\
\text { (b) }\|\bar{c}-c\|_{0, \infty} \leq M h_{c}^{l+1}\left(\log h_{c}^{-1}\right)^{r}\|c\|_{l+1, \infty},
\end{gathered}
$$

where

$$
r= \begin{cases}0 & \text { when } l>1 \\ 1 & \text { when } l=1\end{cases}
$$

Using the inverse relations on $M_{h}$, we know for $z \in M_{h}$ that

$$
\begin{aligned}
\left\|\nabla \frac{\partial}{\partial t} \bar{c}\right\|_{0, \infty} \leq & \left\|\nabla \frac{\partial}{\partial t}(\bar{c}-z)\right\|_{0, \infty}+\left\|\nabla \frac{\partial}{\partial t}(c-z)\right\|_{0, \infty}+\left\|\nabla \frac{\partial}{\partial t} c\right\|_{0, \infty} \\
\leq & K h_{c}^{-2}\left\|\frac{\partial}{\partial t}(\bar{c}-z)\right\|+\left\|\nabla \frac{\partial}{\partial t}(c-z)\right\|_{0, \infty}+\left\|\nabla \frac{\partial}{\partial t} c\right\|_{0, \infty} \\
\leq & K h_{c}^{-2}\left(\left\|\frac{\partial}{\partial t}(\bar{c}-c)\right\|+\left\|\frac{\partial}{\partial t}(c-z)\right\|\right)+\left\|\nabla \frac{\partial}{\partial t}(c-z)\right\|_{0, \infty} \\
& +\left\|\nabla \frac{\partial}{\partial t} c\right\|_{0, \infty} .
\end{aligned}
$$

Choosing $z$ as the interpolant of $c$ in $M_{h}$, we see from the above inequality that

$$
\left\|\nabla \frac{\partial}{\partial t} \bar{c}\right\|_{0, \infty} \leq K h_{c}^{l-1}\left(\|c\|_{l+1}+\left\|\frac{\partial c}{\partial t}\right\|_{l+1}+h_{c}^{l}\left\|\frac{\partial}{\partial t} c\right\|_{l+1, \infty}\right)+\left\|\nabla \frac{\partial}{\partial t} c\right\|_{0, \infty} .
$$

This shows that $\left\|\nabla \frac{\partial}{\partial t} \bar{c}\right\|_{0, \infty}$ is bounded. In a similar fashion one can show that $\|\bar{c}\|_{1, \infty}$ and $\left\|\frac{\partial}{\partial t} \bar{c}\right\|_{0, \infty}$ are bounded. 
Write $\xi=C-\bar{c}, \zeta=\bar{c}-c$, subtract (2.5) from (2.10a), and apply (3.1) to obtain

$$
\begin{aligned}
&\left(\varphi \frac{\partial \xi}{\partial t}, z\right)+(D \nabla \xi, \nabla z) \\
&=(f(C)-f(c), z)-\left(\varphi \frac{\partial \zeta}{\partial t}, z\right)-(b(C) U-b(c) u, \nabla z) \\
&-\left((b(C)-b(c)) u_{0}, \nabla z\right)-(e(C) U \cdot \nabla C-e(c) u \cdot \nabla \bar{c}, z) \\
&-\left((e(C) \nabla C-e(c) \nabla \bar{c}) \cdot u_{0}, z\right) \\
&-(A(C) \cdot U-A(c) \cdot u, z)+\lambda(\zeta, z), \quad z \in M_{h} .
\end{aligned}
$$

Differentiate equation (3.3) to show that

$$
\begin{aligned}
\left(\varphi \frac{\partial^{2} \xi}{\partial t^{2}}, z\right)+\left(D \nabla \frac{\partial \xi}{\partial t}, \nabla z\right) \\
=\left(\frac{\partial}{\partial t}(f(C)-f(c)), z\right)-\left(\varphi \frac{\partial^{2} \zeta}{\partial t^{2}}, z\right) \\
\quad-\left(\frac{\partial}{\partial t}(b(C) U-b(c) u), \nabla z\right) \\
\quad-\left(\frac{\partial}{\partial t}(b(C)-b(c)) u_{0}, \nabla z\right) \\
\quad-\left(\frac{\partial}{\partial t}(e(C) U \cdot \nabla C-e(c) u \cdot \nabla \bar{c}), z\right) \\
\quad-\left(\frac{\partial}{\partial t}(e(C) \nabla C-e(c) \nabla \bar{c}) \cdot u_{0}, z\right) \\
\quad-\left(\frac{\partial}{\partial t}(A(C) \cdot U-A(c) \cdot u), z\right)+\lambda\left(\frac{\partial \zeta}{\partial t}, z\right), \quad z \in M_{h} .
\end{aligned}
$$

By selecting the test function $z=\frac{\partial \xi}{\partial t}$, and using the boundedness of $\|\bar{c}\|_{0, \infty}$ and $\left\|\frac{\partial \tau}{\partial t}\right\|_{1, \infty}$, it follows from (3.2) that

$$
\begin{aligned}
\frac{1}{2} \frac{d}{d t}( & \left.\varphi \frac{\partial \xi}{\partial t}, \frac{\partial \xi}{\partial t}\right)+\left(D \nabla \frac{\partial \xi}{\partial t}, \nabla \frac{\partial \xi}{\partial t}\right) \\
\leq & K\left\{\|\xi\|^{2}+\left\|\frac{\partial \xi}{\partial t}\right\|^{2}+h_{c}^{2 l+2}\right\}-\left(\frac{\partial}{\partial t}(b(C) U-b(c) u), \nabla \frac{\partial \xi}{\partial t}\right) \\
& -\left(\frac{\partial}{\partial t}(b(C)-b(c)) u_{0}, \nabla \frac{\partial \xi}{\partial t}\right) \\
& -\left(\frac{\partial}{\partial t}(e(C) U \cdot \nabla C-e(c) u \cdot \nabla \bar{c}), \frac{\partial \xi}{\partial t}\right) \\
& -\left(\frac{\partial}{\partial t}(e(C) \nabla C-e(c) \nabla \bar{c}) \cdot u_{0}, \frac{\partial \xi}{\partial t}\right) \\
& -\left(\frac{\partial}{\partial t}(A(C) \cdot U-A(c) \cdot u), \frac{\partial \xi}{\partial t}\right) .
\end{aligned}
$$


Next, observe from (3.2) and the inequality $a b \leq \varepsilon a^{2}+b^{2} / 4 \varepsilon$ that

$$
\begin{aligned}
& \left|\left(\frac{\partial}{\partial t}(b(C) U-b(c) u), \nabla \frac{\partial \xi}{\partial t}\right)\right| \\
& =\mid\left(\frac{\partial b}{\partial c}(C) \frac{\partial \xi}{\partial t}(U-u), \nabla \frac{\partial \xi}{\partial t}\right) \\
& +\left(\frac{\partial b}{\partial c}(C) \frac{\partial \bar{c}}{\partial t}(U-u)+\frac{\partial b}{\partial c}(C) \frac{\partial(\xi+\zeta)}{\partial t} u\right. \\
& +\left(\frac{\partial b}{\partial c}(C)-\frac{\partial b}{\partial c}(c)\right) \frac{\partial c}{\partial t} u+b(C) \frac{\partial(U-u)}{\partial t} \\
& \left.+(b(C)-b(c)) \frac{\partial u}{\partial t}, \nabla \frac{\partial \xi}{\partial t}\right) \mid \\
& \leq K\left\|\nabla \frac{\partial \xi}{\partial t}\right\|\left\{\left(\left\|\frac{\partial \xi}{\partial t}\right\|_{0, \infty}+1\right)\|U-u\|\right. \\
& \left.+\|\xi\|+\left\|\frac{\partial \xi}{\partial t}\right\|+\left\|\frac{\partial(U-u)}{\partial t}\right\|+h_{c}^{l+1}\right\} \\
& \leq \varepsilon\left\|\nabla \frac{\partial \xi}{\partial t}\right\|^{2}+K\left\{\|\xi\|^{2}+\left\|\frac{\partial \xi}{\partial t}\right\|^{2}+\left(\left\|\frac{\partial \xi}{\partial t}\right\|_{0, \infty}^{2}+1\right)\|U-u\|^{2}\right. \\
& \left.+\left\|\frac{\partial(U-u)}{\partial t}\right\|^{2}+h_{c}^{2 l+2}\right\} .
\end{aligned}
$$

Similarly,

$$
\begin{aligned}
& \left(\frac{\partial}{\partial t}(e(C) U \cdot \nabla C-e(c) u \cdot \nabla \bar{c}), \frac{\partial \xi}{\partial t}\right) \\
& =\left(\frac{\partial e}{\partial c}(C) \frac{\partial C}{\partial t}(U-u) \cdot \nabla C+\frac{\partial e}{\partial c}(C) \frac{\partial C}{\partial t} u \cdot \nabla \xi\right. \\
& \left.\quad+\frac{\partial e}{\partial c}(C) \frac{\partial(\xi+\zeta)}{\partial t} u \cdot \nabla \bar{c}+\left(\frac{\partial e}{\partial c}(C)-\frac{\partial e}{\partial c}(c)\right) \frac{\partial c}{\partial t} u \cdot \nabla \bar{c}, \frac{\partial \xi}{\partial t}\right) \\
& +\left(e(C) U \cdot \nabla \frac{\partial \xi}{\partial t}+e(C)(U-u) \cdot \nabla \frac{\partial \bar{c}}{\partial t}\right. \\
& \left.\quad+(e(C)-e(c)) u \cdot \nabla \frac{\partial \bar{c}}{\partial t}, \frac{\partial \xi}{\partial t}\right) \\
& +\left(e(C) \frac{\partial(U-u)}{\partial t} \cdot \nabla C+e(C) \frac{\partial u}{\partial t} \cdot \nabla \xi\right. \\
& \left.+(e(C)-e(c)) \frac{\partial u}{\partial t} \cdot \nabla \bar{c}, \frac{\partial \xi}{\partial t}\right)
\end{aligned}
$$


where

$$
\begin{aligned}
\left|\left(\frac{\partial e}{\partial c}(C) \frac{\partial C}{\partial t}(U-u) \cdot \nabla C, \frac{\partial \xi}{\partial t}\right)\right| & \\
\leq & \left|\left(\frac{\partial e}{\partial c}(C) \frac{\partial \xi}{\partial t}(U-u) \cdot \nabla \xi, \frac{\partial \xi}{\partial t}\right)\right|+\left|\left(\frac{\partial e}{\partial c}(C) \frac{\partial \bar{c}}{\partial t}(U-u) \cdot \nabla \xi, \frac{\partial \xi}{\partial t}\right)\right| \\
& +\left|\left(\frac{\partial e}{\partial c}(C) \frac{\partial \xi}{\partial t}(U-u) \cdot \nabla \bar{c}, \frac{\partial \xi}{\partial t}\right)\right| \\
& +\left|\left(\frac{\partial e}{\partial c}(C) \frac{\partial \bar{c}}{\partial t}(U-u) \cdot \nabla \bar{c}, \frac{\partial \xi}{\partial t}\right)\right| \\
\leq & K\|U-u\|\left\{\left\|\frac{\partial \xi}{\partial t}\right\|_{0, \infty}^{2}\|\nabla \xi\|+\left\|\frac{\partial \xi}{\partial t}\right\|_{0, \infty}\left(\|\nabla \xi\|+\left\|\frac{\partial \xi}{\partial t}\right\|\right)+\left\|\frac{\partial \xi}{\partial t}\right\|\right\} \\
\leq & K\left(\left\|\frac{\partial \xi}{\partial t}\right\|_{0, \infty}^{2}+1\right)\left\{\|\nabla \xi\|^{2}+\left\|\frac{\partial \xi}{\partial t}\right\|^{2}+\|U-u\|^{2}\right\},
\end{aligned}
$$

$$
\begin{gathered}
\left|\left(\frac{\partial e}{\partial c}(C) \frac{\partial C}{\partial t} u \cdot \nabla \xi, \frac{\partial \xi}{\partial t}\right)\right| \leq K\left(\left\|\frac{\partial \xi}{\partial t}\right\|_{0, \infty}^{2}+1\right)\left\{\|\nabla \xi\|^{2}+\left\|\frac{\partial \xi}{\partial t}\right\|^{2}\right\} \\
\left|\left(e(C) U \cdot \nabla \frac{\partial \xi}{\partial t}, \frac{\partial \xi}{\partial t}\right)\right| \leq K\left\|\nabla \frac{\partial \xi}{\partial t}\right\|\left\{\left\|\frac{\partial \xi}{\partial t}\right\|_{0, \infty} \cdot\|U-u\|+\left\|\frac{\partial \xi}{\partial t}\right\|\right\} \\
\leq \varepsilon\left\|\nabla \frac{\partial \xi}{\partial t}\right\|^{2}+K\left\{\left\|\frac{\partial \xi}{\partial t}\right\|^{2}+\left\|\frac{\partial \xi}{\partial t}\right\|_{0, \infty}^{2}\|U-u\|^{2}\right\}, \\
\left|\left(e(C) \frac{\partial(U-u)}{\partial t} \cdot \nabla C, \frac{\partial \xi}{\partial t}\right)\right| \\
\leq K\left\|\frac{\partial(U-u)}{\partial t}\right\|\left\{\left\|\frac{\partial \xi}{\partial t}\right\|_{0, \infty}\|\nabla \xi\|+\left\|\frac{\partial \xi}{\partial t}\right\|\right\} \\
\leq K\left\{\left\|\frac{\partial \xi}{\partial t}\right\|_{0, \infty}^{2}\|\nabla \xi\|^{2}+\left\|\frac{\partial \xi}{\partial t}\right\|^{2}+\left\|\frac{\partial(U-u)}{\partial t}\right\|^{2}\right\}
\end{gathered}
$$

The remaining terms of (3.7) may be treated the same way as above. Hence,

$$
\begin{aligned}
&\left|\left(\frac{\partial}{\partial t}(e(C) U \cdot \nabla C-e(c) u-\nabla \bar{c}), \frac{\partial \xi}{\partial t}\right)\right| \\
& \leq \varepsilon\left\|\nabla \frac{\partial \xi}{\partial t}\right\|^{2}+K\left\{\|\xi\|^{2}+\left(\left\|\frac{\partial \xi}{\partial t}\right\|_{0, \infty}^{2}+1\right)\right. \\
& \cdot\left(\|\nabla \xi\|^{2}+\left\|\frac{\partial \xi}{\partial t}\right\|^{2}+\|U-u\|^{2}\right) \\
&\left.+\left\|\frac{\partial(U-u)}{\partial t}\right\|^{2}\right\} K h_{c}^{2 l+2} .
\end{aligned}
$$


An argument similar to that used above can be applied to the remaining terms in (3.5) to show that

$$
\begin{aligned}
& \frac{1}{2} \frac{d}{d t}\left(\varphi \frac{\partial \xi}{\partial t}, \frac{\partial \xi}{\partial t}\right)+\frac{1}{2} D_{*}\left\|\nabla \frac{\partial \xi}{\partial t}\right\|^{2} \\
& \leq K\left\{\|\xi\|^{2}+\left(\left\|\frac{\partial \xi}{\partial t}\right\|_{0, \infty}^{2}+1\right)\left(\|\nabla \xi\|^{2}+\left\|\frac{\partial \xi}{\partial t}\right\|^{2}+\|U-u\|^{2}\right)\right. \\
&\left.+\left\|\frac{\partial}{\partial t}(U-u)\right\|^{2}+h_{c}^{2 l+2}\right\}
\end{aligned}
$$

where $D_{*}$ is a lower bound of $D$. Integrate the inequality (3.13) with respect to time $t$ to show that

$$
\begin{aligned}
&\left\|\frac{\partial \xi}{\partial t}\right\|^{2}+\int_{0}^{t}\left\|\nabla \frac{\partial \xi}{\partial t}\right\|^{2} d s \\
& \leq K\left\{\left\|\frac{\partial \xi}{\partial t}(0)\right\|^{2}+\int_{0}^{t}\left[\|\xi\|^{2}+\left(\left\|\frac{\partial \xi}{\partial t}\right\|_{0, \infty}^{2}+1\right)\right.\right. \\
& \cdot\left(\|\nabla \xi\|^{2}+\left\|\frac{\partial \xi}{\partial t}\right\|^{2}+\|U-u\|^{2}\right) \\
&\left.\left.+\left\|\frac{\partial}{\partial t}(U-u)\right\|^{2}\right] d s+h_{c}^{2 l+2}\right\} .
\end{aligned}
$$

Set $t=0$ in (2.5) and (2.10a) and use (2.7), (2.10c), and (2.12) to obtain

$$
\left(\varphi \frac{\partial}{\partial t}(C-c)(0), z\right)=0, \quad z \in M_{h}
$$

Let $z=\frac{\partial \xi}{\partial t}(0)$ in $(3.15)$; then

$$
\left\|\frac{\partial \xi}{\partial t}(0)\right\| \leq K\left\|\frac{\partial \zeta}{\partial t}(0)\right\| \leq K h_{c}^{l+1}
$$

Adding the following inequalities

$$
\begin{gathered}
\|\xi\|^{2}=\int_{0}^{t} \frac{d}{d t}\|\xi\|^{2} d s \leq \int_{0}^{t}\left[\|\xi\|^{2}+\left\|\frac{\partial \xi}{\partial t}\right\|^{2}\right] d s \\
\|\nabla \xi\|^{2}=\int_{0}^{t} \frac{d}{d t}\|\nabla \xi\|^{2} d s \leq \varepsilon \int_{0}^{t}\left\|\nabla \frac{\partial \xi}{\partial t}\right\|^{2} d s+K \int_{0}^{t}\|\nabla \xi\|^{2} d s
\end{gathered}
$$


to $(3.14)$, we obtain

$$
\begin{aligned}
&\|\xi\|_{1}^{2}+\left\|\frac{\partial \xi}{\partial t}\right\|^{2}+\int_{0}^{t}\left\|\nabla \frac{\partial \xi}{\partial t}\right\|^{2} d s \\
& \leq K_{1}\left\{\int _ { 0 } ^ { t } \left[\|\xi\|^{2}+\left(\left\|\frac{\partial \xi}{\partial t}\right\|_{0, \infty}^{2}+1\right)\right.\right. \\
& \cdot\left(\|\nabla \xi\|^{2}+\left\|\frac{\partial \xi}{\partial t}\right\|^{2}+\|U-u\|^{2}\right) \\
&\left.\left.+\left\|\frac{\partial}{\partial t}(U-u)\right\|^{2}\right] d s+h_{c}^{2 l+2}\right\},
\end{aligned}
$$

where

$$
K_{1}=K_{1}\left(\|\bar{c}\|_{1, \infty},\left\|\frac{\partial \bar{c}}{\partial t}\right\|_{1, \infty},\|c\|_{l+1},\left\|\frac{\partial c}{\partial t}\right\|_{l+1},\left\|\frac{\partial^{2} c}{\partial t^{2}}\right\|_{l+1}\right)
$$

\section{ERROR ESTIMATES FOR THE PRESSURE EQUATION}

In this section we first give error estimates for the pressure equation. These estimates are then combined with the estimates obtained in $\S 3$ to yield the main theorem of the paper.

To estimate the errors in the approximation of the pressure equation, we again introduce an elliptic projection in the sense of Johnson and Thomé [6]. Let $(\bar{u}, \bar{p}) \in V_{h} \times W_{h}$ be the solution of the system

$$
\begin{array}{ll}
\text { (a) }(\nabla \cdot(\bar{u}-u), w)+(\bar{p}-p, w)=0, & w \in W_{h}, \\
\text { (b) }(\alpha(c)(\bar{u}-u), v)-(\nabla \cdot v, \bar{p}-p)=0, & v \in V_{h}, \\
\text { (c) }(\bar{p}, 1)=(p, 1), &
\end{array}
$$

where $(c, p, u)$ is the solution of $(2.5)-(2.7)$.

To show the uniqueness and existence of the solution of (4.1), it suffices to show that the associated homogeneous system has only the trivial solution. The associated homogeneous system is

$$
\begin{array}{lll}
\text { (a) } & (\nabla \cdot \bar{u}, w)+(\bar{p}, w)=0, & w \in W_{h}, \\
\text { (b) }(\alpha(c) \bar{u}, v)-(\nabla \cdot v, \bar{p})=0, & v \in V_{h} .
\end{array}
$$

Choosing $w=\bar{p}$ and $v=\bar{u}$ in (4.2) leads to

$$
0 \leq \alpha_{*}(\bar{u}, \bar{u}) \leq(\alpha(c) \bar{u}, \bar{u})=(\nabla \cdot \bar{u}, \bar{p})=-(\bar{p}, \bar{p}) \leq 0,
$$

where $\alpha_{*}$ is a positive number such that $\alpha_{*} \leq \alpha(c)$. Hence, $\bar{u}=0$ and $\bar{p}=0$.

In the analysis to follow we shall employ three linear operators: the $L^{2}$ orthogonal projections $r_{h_{c}}: H^{1}(\Omega) \rightarrow M_{h}$ and $r_{h_{p}}: W \rightarrow W_{h}$, respectively defined by
(a) $\left(\varphi-r_{h_{c}} \varphi, m\right)=0$,
$m \in M_{h}$,
(b) $\left(\varphi-r_{h_{p}} \varphi, w\right)=0$,
$w \in W_{h}$, 
and the linear operator $T_{h}: V \rightarrow V_{h}$ having the property

$$
\left(\nabla \cdot\left(v-T_{h} v\right), w\right)=0, \quad w \in W_{h} .
$$

From [6] the following results are standard:
(a) $\left\|\varphi-r_{h_{c}} \varphi\right\| \leq M h_{c}^{l+1}\|\varphi\|_{l+1}$, $l \geq 1$,
(b) $\left\|\varphi-r_{h_{p}} \varphi\right\| \leq M h_{p}^{k+1}\|\varphi\|_{k+1}$, $k \geq 0$,
(c) $\left\|\varphi-r_{h_{p}} \varphi\right\|_{0, \infty} \leq M h_{p}^{k+1}\left(\log h_{p}^{-1}\right)^{1 / 2}\|\varphi\|_{k+2}, \quad k \geq 0$,
(d) $\left\|v-T_{h} v\right\| \leq M h_{p}^{k+1}\|v\|_{k+1}$, $k \geq 0$.

Choosing $w=\bar{p}-r_{h_{p}} p$ and $v=\bar{u}-T_{h} u$ in (4.1) and adding the two resulting equations, we have

$$
\begin{aligned}
& \left(\alpha(c)(\bar{u}-u), \bar{u}-T_{h} u\right)-\left(\nabla \cdot\left(\bar{u}-T_{h} u\right), \bar{p}-p\right) \\
& \quad+\left(\nabla \cdot(\bar{u}-u), \bar{p}-r_{h_{p}} p\right)+\left(\bar{p}-p, \bar{p}-r_{h_{p}} p\right)=0
\end{aligned}
$$

Use the properties of $r_{h_{p}}$ and $T_{h}$ to show that

$$
\begin{aligned}
& \left(\nabla \cdot\left(\bar{u}-T_{h} u\right), \bar{p}-p\right)-\left(\nabla \cdot(\bar{u}-u), \bar{p}-r_{h} p\right) \\
& \quad=\left(\nabla \cdot\left(\bar{u}-T_{h} u\right), \bar{p}-r_{h_{p}} p\right)-\left(\nabla \cdot\left(\bar{u}-T_{h} u\right), \bar{p}-r_{h} p\right)=0 .
\end{aligned}
$$

Then from (4.7),

$$
\begin{aligned}
& (\alpha(c)(\bar{u}-u), \bar{u}-u)+(\bar{p}-p, \bar{p}-p) \\
& \quad=\left(\alpha(c)(\bar{u}-u), T_{h} u-u\right)+\left(\bar{p}-p, r_{h_{p}} p-p\right) \\
& \quad \leq \frac{\alpha_{*}}{2}\|\bar{u}-u\|^{2}+K\left\|T_{h} u-u\right\|^{2}+\frac{1}{2}\|\bar{p}-p\|^{2}+\frac{1}{2}\left\|r_{h_{p}} p-p\right\|^{2},
\end{aligned}
$$

and from (4.6),

$$
\|\bar{u}-u\|+\|\bar{p}-p\| \leq K h_{p}^{k+1}\left\{\|u\|_{k+1}+\|p\|_{k+1}\right\}, \quad k \geq 0 .
$$

Now we derive the $L^{\infty}$-error estimates. By (4.1b) and (4.4),

$$
(\alpha(c)(\bar{u}-u), v)+\left(r_{h_{p}} p-\bar{p}, \nabla \cdot v\right)=0, \quad v \in V_{h} .
$$

Thus, from [6, Lemma 1.2] and (4.8) we have

$$
\begin{aligned}
\left\|r_{h_{p}} p-\bar{p}\right\|_{0, \infty} & \leq K \log h_{p}^{-1}\|\alpha(c)(\bar{u}-u)\| \\
& \leq K h_{p}^{k+1} \log h_{p}^{-1}\left\{\|u\|_{k+1}+\|p\|_{k+1}\right\}
\end{aligned}
$$


Consequently, from (4.6c)

$$
\begin{aligned}
\|\bar{p}-p\|_{0, \infty} & \leq\left\|\bar{p}-r_{h_{p}} p\right\|_{0, \infty}+\left\|r_{h_{p}} p-p\right\|_{0, \infty} \\
& \leq K h_{p}^{k+1} \log h_{p}^{-1}\left\{\|u\|_{k+1}+\|p\|_{k+2}\right\}, \quad k \geq 0 .
\end{aligned}
$$

As for the $L^{2}$-estimates for $\frac{\partial}{\partial t}(\bar{u}-u)$ and $\frac{\partial}{\partial t}(\bar{p}-p)$, we differentiate (4.1) in time to show that

$$
\begin{gathered}
\text { (a) }\left(\nabla \cdot \frac{\partial}{\partial t}(\bar{u}-u), w\right)+\left(\frac{\partial}{\partial t}(\bar{p}-p), w\right)=0, \quad w \in W_{h}, \\
\text { (b) }\left(\alpha(c) \frac{\partial}{\partial t}(\bar{u}-u), v\right)+\left(\frac{\partial \alpha}{\partial c}(c) \frac{\partial c}{\partial t}(\bar{u}-u), v\right) \\
-\left(\nabla \cdot v, \frac{\partial}{\partial t}(\bar{p}-p)\right)=0, \quad v \in V_{h} .
\end{gathered}
$$

Select $w=\frac{\partial \tilde{p}}{\partial t}-r_{h_{p}} \frac{\partial p}{\partial t}$ and $v=\frac{\partial u}{\partial t}-T_{h} \frac{\partial u}{\partial t}$ in (4.12) and proceed as in deriving (4.8) to obtain

$$
\begin{aligned}
(\alpha(c) & \left.\frac{\partial}{\partial t}(\bar{u}-u), \frac{\partial}{\partial t}(\bar{u}-u)\right)+\left(\frac{\partial}{\partial t}(\bar{p}-p), \frac{\partial}{\partial t}(\bar{p}-p)\right) \\
= & \left(\alpha(c) \frac{\partial}{\partial t}(\bar{u}-u), T_{h} \frac{\partial u}{\partial t}-\frac{\partial u}{\partial t}\right)-\left(\frac{\partial \alpha}{\partial c}(c) \frac{\partial c}{\partial t}(\bar{u}-u), \frac{\partial}{\partial t}(\bar{u}-u)\right) \\
& -\left(\frac{\partial \alpha}{\partial c}(c) \frac{\partial c}{\partial t}(\bar{u}-u), \frac{\partial u}{\partial t}-T_{h} \frac{\partial u}{\partial t}\right)+\left(\frac{\partial}{\partial t}(\bar{p}-p), r_{h_{p}} \frac{\partial p}{\partial t}-\frac{\partial p}{\partial t}\right) \\
\leq & \frac{1}{2} \alpha_{*}\left\|\frac{\partial}{\partial t}(\bar{u}-u)\right\|^{2}+\frac{1}{2}\left\|\frac{\partial}{\partial t}(\bar{p}-p)\right\|^{2} \\
& +K\left\{\left\|\frac{\partial u}{\partial t}-T_{h} \frac{\partial u}{\partial t}\right\|^{2}+\|\bar{u}-u\|^{2}+\left\|r_{h_{p}} \frac{\partial p}{\partial t}-\frac{\partial p}{\partial t}\right\|^{2}\right\} .
\end{aligned}
$$

From (4.6) and (4.8), we have

$$
\begin{aligned}
& \left\|\frac{\partial}{\partial t}(\bar{u}-u)\right\|+\left\|\frac{\partial}{\partial t}(\bar{p}-p)\right\| \\
& \quad \leq K h_{p}^{k+1}\left\{\|u\|_{k+1}+\|p\|_{k+1}+\left\|\frac{\partial u}{\partial t}\right\|_{k+1}+\left\|\frac{\partial p}{\partial t}\right\|_{k+1}\right\}, \quad k \geq 0 .
\end{aligned}
$$

Similarly,

$$
\begin{aligned}
\left\|\frac{\partial^{2}}{\partial t^{2}}(\bar{p}-p)\right\| \leq K h_{p}^{k+1}\{ & \|u\|_{k+1}+\|p\|_{k+1}+\left\|\frac{\partial u}{\partial t}\right\|_{k+1} \\
& \left.+\left\|\frac{\partial p}{\partial t}\right\|_{k+1}+\left\|\frac{\partial^{2} u}{\partial t^{2}}\right\|_{k+1}+\left\|\frac{\partial^{2} p}{\partial t^{2}}\right\|_{k+1}\right\} .
\end{aligned}
$$


Now we can turn to the estimate for the pressure equation. Let

$$
\pi=P-\bar{p}, \quad \eta=\bar{p}-p, \quad \sigma=U-\bar{u}, \quad \rho=\bar{u}-u .
$$

Combine (2.6), (2.10b), and (4.1) to show that

$$
\begin{aligned}
\text { (a) }(d(C) & \left.\frac{\partial \pi}{\partial t}, w\right)+(\nabla \cdot \sigma, w) \\
= & (\eta, w)-\left(d(C) \frac{\partial \eta}{\partial t}, w\right) \\
& -\left((d(C)-d(c)) \frac{\partial p}{\partial t}, w\right), \quad w \in W_{h}, \\
\text { (b) } \quad(\alpha(C) \sigma, v)-(\nabla \cdot v, \pi) & \\
= & ((\alpha(c)-\alpha(C)) \bar{u}, v)+\left((\alpha(c)-\alpha(C)) u_{0}, v\right), \quad v \in V_{h} .
\end{aligned}
$$

Differentiate (4.16) with respect to time to show that

$$
\begin{aligned}
(d(C) & \left.\frac{\partial^{2} \pi}{\partial t^{2}}, w\right)+\left(\nabla \cdot \frac{\partial \sigma}{\partial t}, w\right) \\
= & \left(\frac{\partial \eta}{\partial t}, w\right)-\left(\frac{\partial d}{\partial c}(C) \frac{\partial C}{\partial t} \frac{\partial \pi}{\partial t}, w\right) \\
& -\left(d(C) \frac{\partial^{2} \eta}{\partial t^{2}}, w\right)-\left(\frac{\partial d}{\partial c}(C) \frac{\partial C}{\partial t} \frac{\partial \eta}{\partial t}, w\right) \\
& -\left(\frac{\partial}{\partial t}[d(C)-d(c)] \frac{\partial p}{\partial t}, w\right) \\
& -\left([d(C)-d(c)] \frac{\partial^{2} p}{\partial t^{2}}, w\right), \quad w \in W_{h}, \\
(\text { b) } \quad(\alpha(C) & \left.\frac{\partial \sigma}{\partial t}, v\right)-\left(\nabla \cdot v, \frac{\partial \pi}{\partial t}\right) \\
= & \left(\frac{\partial}{\partial t}[\alpha(c)-\alpha(C)] \bar{u}, v\right)+\left([\alpha(c)-\alpha(C)] \frac{\partial \bar{u}}{\partial t}, v\right) \\
& +\left(\frac{\partial}{\partial t}[\alpha(c)-\alpha(C)] u_{0}, v\right) \\
& -\left(\frac{\partial \alpha}{\partial c}(C) \frac{\partial C}{\partial t} \sigma, v\right), \quad v \in V_{h} .
\end{aligned}
$$

Select the test functions $w=\frac{\partial \pi}{\partial t}$ and $v=\frac{\partial \sigma}{\partial t}$ in (4.17), add the two equations, and use the equality

$$
\frac{d}{d t}\left(d(C) \frac{\partial \pi}{\partial t}, \frac{\partial \pi}{\partial t}\right)=2\left(d(C) \frac{\partial^{2} \pi}{\partial t^{2}}, \frac{\partial \pi}{\partial t}\right)+\left(\frac{\partial d}{\partial c}(C) \frac{\partial C \partial \pi}{\partial t \partial t}, \frac{\partial \pi}{\partial t}\right)
$$


to see that

$$
\begin{aligned}
\frac{1}{2} \frac{d}{d t}( & \left.d(C) \frac{\partial \pi}{\partial t}, \frac{\partial \pi}{\partial t}\right)+\left(\alpha(C) \frac{\partial \sigma}{\partial t}, \frac{\partial \sigma}{\partial t}\right) \\
= & \left(\frac{\partial \eta}{\partial t}, \frac{\partial \pi}{\partial t}\right)-\frac{1}{2}\left(\frac{\partial d}{\partial c}(C) \frac{\partial C \partial \pi}{\partial t \partial t}, \frac{\partial \pi}{\partial t}\right)-\left(d(C) \frac{\partial^{2} \eta}{\partial t^{2}}, \frac{\partial \pi}{\partial t}\right) \\
& -\left(\frac{\partial d}{\partial c}(C) \frac{\partial C \partial \eta}{\partial t \partial t}, \frac{\partial \pi}{\partial t}\right)-\left(\frac{\partial}{\partial t}[d(C)-d(c)] \frac{\partial p}{\partial t}, \frac{\partial \pi}{\partial t}\right) \\
& -\left([d(C)-d(c)] \frac{\partial^{2} p}{\partial t^{2}}, \frac{\partial \pi}{\partial t}\right)+\left(\frac{\partial}{\partial t}[\alpha(c)-\alpha(C)] \bar{u}, \frac{\partial \sigma}{\partial t}\right) \\
& +\left([\alpha(c)-\alpha(C)] \frac{\partial \bar{u}}{\partial t}, \frac{\partial \sigma}{\partial t}\right)+\left(\frac{\partial}{\partial t}[\alpha(c)-\alpha(C)] u_{0}, \frac{\partial \sigma}{\partial t}\right) \\
& -\left(\frac{\partial \alpha}{\partial c}(C) \frac{\partial C}{\partial t} \sigma, \frac{\partial \sigma}{\partial t}\right) \\
\leq & \left\|\frac{\partial \sigma}{\partial t}\right\|^{2}+K\left\{\left\|\frac{\partial \pi}{\partial t}\right\|^{2}+\|\xi\|^{2}+\left\|\frac{\partial \xi}{\partial t}\right\|^{2}+\left\|\frac{\partial \eta}{\partial t}\right\|^{2}\right. \\
& -\left(\frac{\partial \alpha}{\partial c}(C) \frac{\partial C}{\partial t} \sigma, \frac{\partial \sigma}{\partial t}\right) \cdot \\
& -\frac{1}{2}\left(\frac{\partial d}{\partial c}(C) \frac{\partial C \partial \pi}{\partial t \partial t}, \frac{\partial \pi}{\partial t}\right)-\left(\frac{\partial d}{\partial c}(C) \frac{\partial C \partial \eta}{\partial t \partial t}, \frac{\partial \pi}{\partial t}\right) \\
& \left(\left\|\frac{\partial^{2} \eta}{\partial t^{2}}\right\|^{2}+\|\zeta\|^{2}+\left\|\frac{\partial \zeta}{\partial t}\right\|^{2}\right\} \\
& (\alpha)
\end{aligned}
$$

Note that by the triangle inequality

$$
\left\|\frac{\partial C}{\partial t}\right\|_{0, \infty} \leq\left\|\frac{\partial \xi}{\partial t}\right\|_{0, \infty}+\left\|\frac{\partial \bar{c}}{\partial t}\right\|_{0, \infty}
$$

By the boundedness of $\left\|\frac{\partial \tau}{\partial t}\right\|_{0, \infty}$ and the known estimates of $\eta$ and $\xi$, it follows from (4.18) that

$$
\begin{gathered}
\frac{1}{2} \frac{d}{d t}\left(d(C) \frac{\partial \pi}{\partial t}, \frac{\partial \pi}{\partial t}\right)+\alpha_{*}\left\|\frac{\partial \sigma}{\partial t}\right\|^{2} \\
\leq \varepsilon\left\|\frac{\partial \sigma}{\partial t}\right\|^{2}+K\left\{\|\xi\|^{2}+\left\|\frac{\partial \xi}{\partial t}\right\|^{2}+\left(\left\|\frac{\partial \xi}{\partial t}\right\|_{0, \infty}^{2}+1\right)\right. \\
\left.\cdot\left(\left\|\frac{\partial \pi}{\partial t}\right\|^{2}+\|\sigma\|^{2}\right)+h_{c}^{2 l+2}+h_{p}^{2 k+2}\right\},
\end{gathered}
$$


which upon integration gives

$$
\begin{aligned}
\left\|\frac{\partial \pi}{\partial t}\right\|^{2}+\int_{0}^{t}\left\|\frac{\partial \sigma}{\partial t}\right\|^{2} d s & \\
\leq K\left\{\left\|\frac{\partial \pi}{\partial t}(0)\right\|^{2}+\int_{0}^{t}\left[\|\xi\|^{2}+\left\|\frac{\partial \xi}{\partial t}\right\|^{2}\right.\right. & +\left(\left\|\frac{\partial \xi}{\partial t}\right\|_{0, \infty}+1\right) \\
& \left.\cdot\left(\left\|\frac{\partial \pi}{\partial t}\right\|^{2}+\|\sigma\|^{2}\right)\right] d s \\
& \left.+h_{c}^{2 l+2}+h_{p}^{2 k+2}\right\} .
\end{aligned}
$$

Choose $t=0$ in (2.6a) and (2.10bi), and subtract the resulting equations, to show that

$$
\left(\alpha(0)\left(\frac{\partial P}{\partial t}-\frac{\partial p}{\partial t}\right)(0), w\right)=0, \quad w \in W_{h},
$$

and

$$
\left\|\frac{\partial \pi}{\partial t}(0)\right\| \leq K\left\|\frac{\partial \eta}{\partial t}(0)\right\| \leq K h_{p}^{k+1} .
$$

Using (2.7), we see from (4.1) that $\bar{p}(0)=0$ and $\bar{u}(0)=0$. Thus, $\pi(0)=0$ and $\sigma(0)=0$. The techniques used in deriving (3.19) from (3.17) and (3.18) can be applied to (4.20) to show that

$$
\begin{aligned}
&\|\pi\|^{2}+\left\|\frac{\partial \pi}{\partial t}\right\|^{2}+\|\sigma\|^{2}+\int_{0}^{t}\left\|\frac{\partial \sigma}{\partial t}\right\|^{2} d s \\
&23) \leq K\left\{\int_{0}^{t}\left[\|\xi\|^{2}+\left\|\frac{\partial \xi}{\partial t}\right\|^{2}+\left(\left\|\frac{\partial \xi}{\partial t}\right\|_{0, \infty}+1\right)\left(\left\|\frac{\partial \pi}{\partial t}\right\|^{2}+\|\sigma\|^{2}\right)\right] d s\right. \\
&\left.+h_{c}^{2 l+2}+h_{p}^{2 k+2}\right\} .
\end{aligned}
$$

Take a $\left(1+K_{1}\right)$-multiple of (4.23), add it to (3.19), and use (4.8) and (4.14) to have

$$
\begin{array}{r}
\|\xi\|_{1}^{2}+\left\|\frac{\partial \xi}{\partial t}\right\|^{2}+\|\pi\|^{2}+\left\|\frac{\partial \pi}{\partial t}\right\|^{2}+\|\sigma\|^{2}+\int_{0}^{t}\left\|\nabla \frac{\partial \xi}{\partial t}\right\|^{2} d s+\int_{0}^{t}\left\|\frac{\partial \sigma}{\partial t}\right\|^{2} d s \\
\leq K\left\{\int _ { 0 } ^ { t } \left[\|\xi\|^{2}+\left(\left\|\frac{\partial \xi}{\partial t}\right\|_{0, \infty}^{2}+1\right)\right.\right. \\
\left.\cdot\left(\|\nabla \xi\|^{2}+\left\|\frac{\partial \xi}{\partial t}\right\|^{2}+\left\|\frac{\partial \pi}{\partial t}\right\|^{2}+\|\sigma\|^{2}+h_{c}^{2 k+2}\right)\right] d s \\
\left.+h_{c}^{2 l+2}+h_{p}^{2 k+2}\right\} .
\end{array}
$$


To estimate (4.24), we make the induction hypothesis that

$$
\begin{gathered}
\left\|\frac{\partial \xi}{\partial t}\right\|_{L^{2}\left(J ; L^{\infty}(\Omega)\right)}\left(\|\nabla \xi\|_{L^{\infty}\left(J ; L^{2}(\Omega)\right)}+\left\|\frac{\partial \xi}{\partial t}\right\|_{L^{\infty}\left(J ; L^{2}(\Omega)\right)}\right. \\
\left.+\left\|\frac{\partial \pi}{\partial t}\right\|_{L_{\left(J ; L^{2}(\Omega)\right)}}+\|\sigma\|_{L^{\infty}\left(J ; L^{2}(\Omega)\right)}+h_{p}^{k+1}\right) \\
\leq K_{2}\left(h_{c}^{l+1}+h_{p}^{k+1}\right), \quad l \geq 1, k \geq 0 .
\end{gathered}
$$

Thus, from Gronwall's lemma we can derive that

$$
\begin{aligned}
& \|\xi\|_{L^{\infty}\left(J ; H^{1}(\Omega)\right)}+\left\|\frac{\partial \xi}{\partial t}\right\|_{L^{\infty}\left(J ; L^{2}(\Omega)\right)}+\|\pi\|_{L^{\infty}\left(J ; L^{2}(\Omega)\right)}+\left\|\frac{\partial \pi}{\partial t}\right\|_{L^{\infty}\left(J ; L^{2}(\Omega)\right)} \\
& +\|\sigma\|_{L^{\infty}\left(J ; L^{2}(\Omega)^{2}\right)}+\left\|\nabla \frac{\partial \xi}{\partial t}\right\|_{L^{2}\left(J ; L^{2}(\Omega)\right)}+\left\|\frac{\partial \sigma}{\partial t}\right\|_{L^{2}\left(J ; L^{2}(\Omega)^{2}\right)} \\
& \leq K_{3}\left(h_{c}^{l+1}+h_{p}^{k+1}\right), \quad l \geq 1, k \geq 0
\end{aligned}
$$

where

$$
\begin{array}{r}
K_{3}=K_{3}\left(K_{1}, K_{2},\|u\|_{k+1},\|p\|_{k+1},\left\|\frac{\partial u}{\partial t}\right\|_{k+1},\right. \\
\left.\left\|\frac{\partial p}{\partial t}\right\|_{k+1},\left\|\frac{\partial^{2} u}{\partial t^{2}}\right\|_{k+1},\left\|\frac{\partial^{2} p}{\partial t^{2}}\right\|_{k+1}\right) .
\end{array}
$$

Now we turn to the justification of the induction hypothesis (4.25). Obviously, (4.25) holds for $t=0$. Since

$$
\begin{aligned}
F(t)=\left\|\frac{\partial \xi}{\partial t}\right\|_{L^{2}\left(0, t ; L^{\infty}(\Omega)\right)}( & \|\nabla \xi\|_{L^{\infty}\left(0, t ; L^{2}(\Omega)\right)}+\left\|\frac{\partial \xi}{\partial t}\right\|_{L^{\infty} 0, t ; L^{2}(\Omega)} \\
+ & \left.\left\|\frac{\partial \pi}{\partial t}\right\|_{L^{\infty}\left(0, t ; L^{2}(\Omega)\right)}+\|\sigma\|_{L^{\infty}\left(0, t ; L^{2}(\Omega)\right)}+h_{p}^{k+1}\right)
\end{aligned}
$$

is a continuous function in $t$, there exists some $t^{*}>0$ such that

$$
\begin{array}{lll}
\text { (a) } & F(t)<K_{2}\left(h_{c}^{l+1}+h_{p}^{k+1}\right), & 0<t<t^{*}, \\
\text { (b) } & F(t)=K_{2}\left(h_{c}^{l+1}+h_{p}^{k+1}\right), & t=t^{*} .
\end{array}
$$

We want to show that $t^{*}=T$. By the well-known imbedding inequality [11] for two space dimensions,

$$
\|v\|_{0, \infty} \leq K_{4}\left(\log h_{c}^{-1}\right)^{1 / 2}\|v\|_{1}, \quad v \in M_{h},
$$


and using (4.26) and (4.27), we can derive

$$
\begin{aligned}
F(t) \leq K_{3} K_{4}\left(\log h_{c}^{-1}\right)^{1 / 2}\left\{4 K_{3} h_{c}^{2 l+2}\right. & +\left(8 K_{3}+1\right) h_{c}^{l+1} h_{p}^{k+1} \\
& \left.+\left(4 K_{3}+1\right) h_{p}^{2 k+2}\right\}, \quad 0 \leq t \leq t^{*} .
\end{aligned}
$$

Suppose that the relation

$$
h_{p}^{2 k+2}\left(\log h_{c}^{-1}\right)^{1 / 2} \rightarrow 0 \quad \text { as } h \rightarrow 0
$$

holds. Then $h$ can be selected to be small enough so that

$$
F\left(t^{*}\right) \leq \frac{K_{2}}{2}\left(h_{c}^{l+1}+h_{p}^{k+1}\right) .
$$

Hence, $t^{*}=T$, and the proof is completed.

The desired $L^{2}$-optimal error estimates now result from the combination of (4.26), (3.2a), (4.8), and (4.14):

$$
\begin{aligned}
\| C-c & \left\|_{L^{\infty}\left(J ; L^{2}(\Omega)\right)}+\right\| \frac{\partial}{\partial t}(C-c)\left\|_{L^{\infty}\left(J ; L^{2}(\Omega)\right)}+\right\| P-p \|_{L^{\infty}\left(J ; L^{2}(\Omega)\right)} \\
& +\left\|\frac{\partial}{\partial t}(P-p)\right\|_{L^{\infty}\left(J ; L^{2}(\Omega)\right)}+\|U-u\|_{L^{\infty}\left(J ; L^{2}(\Omega)^{2}\right)} \\
& +\left\|\frac{\partial}{\partial t}(U-u)\right\|_{L^{2}\left(J ; L^{2}(\Omega)^{2}\right)} \\
\leq & K_{5}\left(h_{c}^{l+1}+h_{p}^{k+1}\right), \quad l \geq 1, \quad k \geq 0
\end{aligned}
$$

where

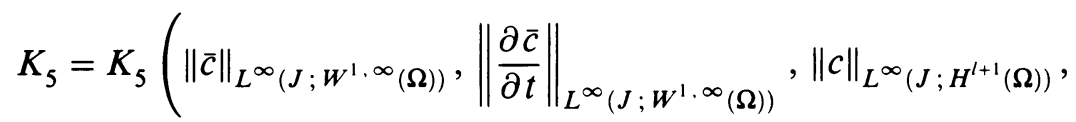

$$
\begin{aligned}
& \left\|\frac{\partial c}{\partial t}\right\|_{L^{\infty}\left(J ; H^{l+1}(\Omega)\right)},\left\|\frac{\partial^{2} c}{\partial t^{2}}\right\|_{L^{2}\left(J ; H^{l+1}(\Omega)\right)},\|p\|_{L^{\infty}\left(J ; H^{k+1}(\Omega)\right)}, \\
& \left\|\frac{\partial p}{\partial t}\right\|_{L^{\infty}\left(J ; H^{k+1}(\Omega)\right)},\left\|\frac{\partial^{2} p}{\partial t^{2}}\right\|_{L^{2}\left(J ; H^{k+1}(\Omega)\right)},\|u\|_{L^{\infty}\left(J ; H^{k+1}(\Omega)\right)}, \\
& \left.\left\|\frac{\partial u}{\partial t}\right\|_{L^{\infty}\left(J ; H^{k+1}(\Omega)\right)},\left\|\frac{\partial^{2} u}{\partial t^{2}}\right\|_{L^{2}\left(J ; H^{k+1}(\Omega)\right)}\right) .
\end{aligned}
$$

Combining (3.2b), (4.26), and (4.29) results in

$$
\begin{aligned}
\|C-c\|_{L^{\infty}\left(J ; L^{\infty}(\Omega)\right)} \leq K_{6}\left(h_{c}^{l+1}+h_{p}^{k+1}\right)\left(\log h_{c}^{-1}\right)^{\beta}, \\
l \geq 1, k \geq 0, \quad \beta= \begin{cases}1, & l=1, \\
\frac{1}{2}, & l \geq 1,\end{cases}
\end{aligned}
$$

where

$$
K_{6}=K_{6}\left(K_{5},\|c\|_{L^{\infty}\left(J ; W^{l+1, \infty}(\Omega)\right)}\right)
$$


Applying a similar argument as in deriving (4.10), we see from (4.16b) that

$$
\begin{aligned}
\|\pi\|_{0, \infty} & \leq K\left\|\alpha(C) \sigma+(\alpha(C)-\alpha(c))\left(\bar{u}+u_{0}\right)\right\| \log h_{p}^{-1} \\
& \leq K\left(h_{c}^{l+1}+h_{p}^{k+1}\right) \log h_{p}^{-1},
\end{aligned}
$$

by (4.26) and (4.32). Then the $L^{\infty}$-estimates for the pressure follows by this estimate and (4.11):

$$
\|P-p\|_{L^{\infty}\left(J ; L^{\infty}(\Omega)\right)} \leq K_{7}\left(h_{c}^{l+1}+h_{p}^{k+1}\right) \log h_{p}^{-1}, \quad l \geq 1, k \geq 0,
$$

where $K_{7}=K_{7}\left(K_{5}\right)$.

Finally, we can return to the error estimates for the original dependent variables $c, p$, and $u$, which are the solution of the system (1.1)-(1.3). The reader is being reminded that the error estimates obtained before pertain to the transformed system whose variables have "*" superscripts. Now define

$$
C=C^{*}+r_{h_{c}} c_{0}, \quad P=P^{*}+r_{h_{p}} p_{0}, \quad U=U^{*}+T_{h} u_{0},
$$

where $\left(C^{*}, P^{*}, U^{*}\right)$ is the solution of the semidiscrete system (2.5)-(2.7). By (2.4), the triangle inequality

$$
\|C-c\| \leq\left\|C^{*}-c^{*}\right\|+\left\|r_{h_{c}} c_{0}-c_{0}\right\|
$$

and (4.32) can be combined with (4.6) to show that

$$
\|C-c\| \leq K\left(h_{c}^{l+1}+h_{p}^{k+1}\right), \quad l \geq 1, k \geq 0 .
$$

By repeated use of (4.6) and (4.32) in the way shown above, we obtain the main theorem of this paper. For the case of using the Raviart-Thomas space of index $k, k \geq 0$, we thus have

Theorem 4.1. Let $(c, p, u)$ be the solution of the continuous problem (1.1)(1.3) and $(C, P, U)$ be defined by (4.35). Suppose that the relation (4.31) on the diameters $h_{p}$ and $h_{c}$ holds. Then the following estimates hold:

(i)

$$
\begin{aligned}
\|C-c\|_{L^{\infty}\left(J ; L^{2}(\Omega)\right)}+\left\|\frac{\partial}{\partial t}(C-c)\right\|_{L^{\infty}\left(J ; L^{2}(\Omega)\right)}+\|P-p\|_{L^{\infty}\left(J ; L^{2}(\Omega)\right)} \\
\quad+\left\|\frac{\partial}{\partial t}(P-p)\right\|_{L^{\infty}\left(J ; L^{2}(\Omega)\right)}+\|U-u\|_{L^{\infty}\left(J ; L^{2}(\Omega)\right)^{2}} \\
\quad+\left\|\frac{\partial}{\partial t}(U-u)\right\|_{L^{2}\left(J ; L^{2}(\Omega)^{2}\right)} \\
\leq K_{8}\left(h_{c}^{l+1}+h_{p}^{k+1}\right), \quad l \geq 1, k \geq 0,
\end{aligned}
$$


where the constant $K_{8}$ depends on

$$
\begin{gathered}
\|\bar{c}\|_{L^{\infty}\left(J ; W^{1, \infty}(\Omega)\right)}, \quad\left\|\frac{\partial \bar{c}}{\partial t}\right\|_{L^{\infty}\left(J ; W^{1, \infty}(\Omega)\right)}, \quad\|c\|_{L^{\infty}\left(J ; H^{l+1}(\Omega)\right)}, \\
\left\|\frac{\partial c}{\partial t}\right\|_{L^{\infty}\left(J ; H^{l+1}(\Omega)\right)}, \quad\left\|\frac{\partial^{2} c}{\partial t^{2}}\right\|_{L^{2}\left(J ; H^{l+1}(\Omega)\right)}, \quad\|p\|_{L^{\infty}\left(J ; H^{k+1}(\Omega)\right)}, \\
\left\|\frac{\partial p}{\partial t}\right\|_{L^{\infty}\left(J ; H^{k+1}(\Omega)\right)}, \quad\left\|\frac{\partial^{2} p}{\partial t^{2}}\right\|_{L^{2}\left(J ; H^{k+1}(\Omega)\right)}, \quad\|u\|_{L^{\infty}\left(J ; H^{k+1}(\Omega)\right)}, \\
\left\|\frac{\partial u}{\partial t}\right\|_{L^{\infty}\left(J ; H^{k+1}(\Omega)\right)}, \quad \text { and }\left\|\frac{\partial^{2} u}{\partial t^{2}}\right\|_{L^{2}\left(J ; H^{k+1}(\Omega)\right)} .
\end{gathered}
$$

(ii)

$$
\begin{aligned}
\|C-c\|_{L^{\infty}\left(J ; L^{\infty}(\Omega)\right)} \leq K_{9}\left(h_{c}^{l+1}+h_{p}^{k+1}\right)\left(\log h_{c}^{-1}\right)^{\beta}, \\
l \geq 1, k \geq 0, \quad \beta= \begin{cases}1, & l=1, \\
\frac{1}{2}, & l>1,\end{cases}
\end{aligned}
$$

where $K_{9}$ depends on $K_{8}$ and $\|c\|_{L^{\infty}\left(J ; W^{l+1, \infty}(\Omega)\right)}$.

(iii)

$$
\|P-p\|_{L^{\infty}\left(J ; L^{\infty}(\Omega)\right)} \leq K_{10}\left(h_{c}^{l+1}+h_{p}^{k+1}\right) \log h_{p}^{-1}, \quad l \geq 1, k \geq 0,
$$

where $K_{10}$ depends on $K_{8}$.

As for the case of the BDM space of index $k, k \geq 1$, replace every occurrence of $k$ by $k-1$ in (4.36)-(4.38).

\section{EXTENSIONS TO SPACE IN THREE DIMENSIONS}

In this section we indicate possible extensions of the analysis in the previous sections to three dimensions. The pressure equation can be approximated using the spaces of $[2,8]$. The error analysis can be carried out in the same way, assuming different relations between the discretization parameters.

Theorem 5.1. Suppose that the mesh parameters satisfy the relations

$$
h_{p}^{2 k+2} h_{c}^{-1 / 2} \rightarrow 0 \text { as } h \rightarrow 0 .
$$

Then the estimate (4.36) holds.

Proof. We follow the proof of Theorem 4.1, making changes when necessary. It suffices to prove that the induction hypothesis (4.25) holds.

Using the imbedding theorem for Sobolev spaces in $R^{3}$, we get

$$
\|v\|_{0,6} \leq K\|v\|_{1}, \quad v \in W^{1,2}(\Omega),
$$

and then, by the inverse inequality in the space $M_{h}$,

$$
\|v\|_{0, \infty} \leq K h^{-1 / 2}\|v\|_{0,6}, \quad v \in M_{h} .
$$


Hence,

$$
\|v\|_{0, \infty} \leq K_{9} h^{-1 / 2}\|v\|_{1}, \quad v \in M_{h} .
$$

It follows from (5.4), (4.26), and (4.27) that

$$
F(t) \leq K_{3} K_{9} h_{c}^{-1 / 2}\left\{4 K_{3} h_{c}^{2 l+2}+\left(8 K_{3}+1\right) h_{c}^{l+1} h_{p}^{k+1}+\left(4 K_{3}+1\right) h_{p}^{2 k+2}\right\} .
$$

By (5.1) and (5.5), the induction hypothesis (4.25) is verified.

As a final remark, we point out that the relation (4.31) or (5.1) in the paper is much weaker than the condition

$$
\min \left(h_{p}^{k+1} h_{c}^{-1}, h_{c}^{l+1} h_{p}^{-1}\right) \rightarrow 0 \text { as } h \rightarrow 0 \text { for all } k,
$$

and

$$
\gamma_{1} \leq h_{p} h_{c}^{-2} \leq \gamma_{2} \text { for } k=0
$$

given in [4].

\section{BIBLIOGRAPHY}

1. F. Brezzi, J. Douglas, Jr., R. Durán, and M. Fortin, Mixed finite elements for second order elliptic problems in three variables, Numer. Math. 51 (1987), 237-250.

2. F. Brezzi, J. Douglas, Jr., M. Fortin, and L. D. Marini, Efficient rectangular mixed finite elements in two and three space variables, RAIRO Modél. Math. Anal. Numér. 21 (1987), 581-604.

3. F. Brezzi, J. Douglas, Jr., and L. D. Marini, Two families of mixed finite elements of second order elliptic problems, Numer. Math. 47 (1985), 217-235.

4. Jim Douglas, Jr. and J. E. Roberts, Numerical methods for a model for compressible miscible displacement in porous media, Math. Comp. 41 (1983), 441-459.

5. C. I. Goldstein and R. Scott, Optimal $L^{\infty}$-estimates for some Galerkin methods for the Dirichlet problem, SIAM J. Numer. Anal. (to appear).

6. C. Johnson and V. Thomée, Error estimates for some mixed finite element methods for parabolic type problems, RAIRO Modél. Math. Anal. Numér. 15 (1981), 41-78.

7. J. A. Nitsche, $L^{\infty}$-convergence of the finite element approximation, Proc. Second Conf. on Finite Elements, Rennes, France, 1975.

8. J. C. Nedelec, Mixed finite elements in $R^{3}$, Numer. Math. 35 (1980), 315-341.

9. __ A new family of mixed finite elements in $R^{3}$, Numer. Math. 50 (1986), 57-82.

10. P. A. Raviart and J. M. Thomas, A mixed finite element method for 2-nd order elliptic problems, Mathematical Aspects of Finite Element Methods, Lecture Notes in Math., vol. 606, Springer-Verlag, 1977, pp. 292-315.

11. A. H. Schatz, V. Thomée, and L. B. Wahlbin, Maximum norm stability and error estimates in parabolic finite element equations, Comm. Pure Appl. Math. 33 (1980), 265-304.

12. R. Scott, Optimal $L^{\infty}$-estimates for the finite element method on irregular meshes, Math. Comp. 30 (1976), 681-697.

13. M. F. Wheeler, A priori $L_{2}$ error estimates for Galerkin approximations to parabolic differential equations, SIAM J. Numer. Anal. 10 (1973), 723-749.

Department of Mathematics and Statistics, Bowling Green State University, BOWLING GREEN, OHIO 43403-0221 